\title{
Spin-orbital physics in the optical conductivity of quarter-filled manganites
}

\author{
Jan Bała ${ }^{1,2}$ and Peter Horsch ${ }^{1}$ \\ ${ }^{1}$ Max-Planck-Institut für Festkörperforschung, Heisenbergstrasse 1, D-70569 Stuttgart, Germany \\ ${ }^{2}$ Marian Smoluchowski Institute of Physics, Jagellonian University, Reymonta 4, PL-30059 Kraków, Poland
}

(Dated: November 3, 2018)

\begin{abstract}
Using finite-temperature diagonalization we investigate the optical conductivity $\sigma(\omega)$ and spinorbital dynamics in the CE phase of half-doped manganites. We find $\sigma(\omega)$ characterized by a broad spectrum with pronounced optical gap due to charge ordering induced by Coulomb and further neighbor Jahn-Teller interactions. With increasing temperature the conductivity shows a significant change over a wide energy range with a characteristic shift towards lower frequencies. In the low temperature CE phase we observe in-gap absorption due to combined orbiton-spin excitations.
\end{abstract}

PACS numbers: 75.10.-b, 71.70.Ej, 75.30.Et, 75.40.Mg

The large variation of physical properties of manganites as function of doping or temperature originates from a complex interplay of spin, orbital and charge degrees of freedom, as well as the interaction with the lattice [1, 2]. An important example for the control of magnetic order due to the orbital degree of freedom is the $\mathrm{CE}$ phase at quarter filling. Here ferromagnetic (FM) zig-zag chains are staggered antiferromagnetically and the occupied $e_{g}$ orbitals are oriented along FM bonds in the $(a, b)$ planes [3] [Fig. 11 (a,b)]. Although the structure reflects the cooperative action of antiferromagnetic (AF) superexchange and FM double-exchange (DE), these interactions alone do not guarantee its stability [4, 5]. The AF-CE phase was observed in cubic $\left.(\mathrm{Nd}, \mathrm{Pr})_{1 / 2}(\mathrm{Sr}, \mathrm{Ca})_{1 / 2} \mathrm{MnO}_{3}, 1\right]$ and in layered $\mathrm{La}_{1 / 2} \mathrm{Sr}_{3 / 2} \mathrm{MnO}_{4}$ [6, 7], $\mathrm{LaSr}_{2} \mathrm{Mn}_{2} \mathrm{O}_{7}$ [8] manganites. Yet CE-type orbital correlations have also been observed in the absence of antiferromagnetism, namely in the FM metallic phase of $\mathrm{Nd}_{1 / 2} \mathrm{Sr}_{1 / 2} \mathrm{MnO}_{3}[9]$, and also at smaller hole doping and are believed to be connected with the mechanism of colossal magnetoresistance [10]. An important feature of the CE-phase is a large optical gap and a broad absorption maximum at 1-2 eV shifting towards lower frequencies with increasing temperature 11, 12, 13]. Remarkably a similar spectral shift is observed in photo-excitation experiments, which reveal ultrafast response times of such Mott insulating structures 2, 14].

Our aim is to investigate the electron dynamics (optical conductivity, elementary excitations) in the CE spinorbital state. It is evident that a real understanding of this phase is only achieved if also the excitation spectra are consistent with experiment. The strong variation of $\sigma(\omega)$ with temperature $T$ was not addressed in previous theoretical work [15, 16]. Our study employing finite temperature diagonalization is designed to explore also quantities, such as the orbital excitation spectra, that so far cannot be measured by experiment and investigate their effect on observables like optical conductivity and spin excitations. Our results for the optical conductivity reproduce for the first time the experimental trends on a semi-quantitative level, and predict orbital excitations in the charge gap of the AF-CE phase.

Orbital degeneracy provides the option for the system to lower its dimensionality, which is essential for the appearance of the CE structure [17]. Our study explores a novel aspect of orbital physics in manganites, namely the role of further neighbor Jahn-Teller (JT) interactions, and the consequences on the dynamics. We find that orbital polarization induced by this interaction is crucial for the appearance of CE-antiferromagnetism at low $\mathrm{T}$, and leads to a spin response consistent with AF-CE order.

We consider an extended DE model with charge order (CO) triggered by Coulomb repulsion and furtherneighbor JT-interaction [5]. AF order along the $c$ axis and $e_{g}$ orbital elongation in the $a / b$ direction confines the electron dynamics to $(a, b)$ planes and thus we consider a two-dimensional (2D) model. Assuming infinite on-site Coulomb repulsion between $e_{g}$ electrons on the same site $(U \rightarrow \infty)$ the model has the form [5]:

$$
\begin{aligned}
H= & -\sum_{\langle\mathbf{i} \mathbf{j}\rangle} \sum_{\xi \zeta, \sigma}\left(t_{\mathbf{i} \mathbf{j}}^{\xi \zeta} \tilde{d}_{\mathbf{i} \xi \sigma}^{\dagger} \tilde{d}_{\mathbf{j} \zeta \sigma}+\text { H.c. }\right)-J_{H} \sum_{\langle\mathbf{i}\rangle} \mathbf{S}_{\mathbf{i}}^{c} \cdot \mathbf{s}_{\mathbf{i}} \\
& +J_{\mathrm{A} F} \sum_{\langle\mathbf{i} \mathbf{j}\rangle} \mathbf{S}_{\mathbf{i}}^{c} \cdot \mathbf{S}_{\mathbf{j}}^{c}+V \sum_{\langle\mathbf{i} \mathbf{j}\rangle} n_{\mathbf{i}} n_{\mathbf{j}}+H_{\mathrm{OO}}
\end{aligned}
$$

The first term describes the motion of $e_{g}$ electrons under the constraint that each site can be occupied at most by one $e_{g}$ electron, $\tilde{d}_{\mathbf{i} \xi \sigma}^{\dagger}=d_{\mathbf{i} \xi \sigma}^{\dagger}\left(1-n_{\mathbf{i} \xi \bar{\sigma}}\right) \prod_{\sigma^{\prime}}\left(1-n_{\mathbf{i} \bar{\xi} \sigma^{\prime}}\right)$. Here the index $\bar{\xi}(\bar{\sigma})$ denotes the $e_{g}$ orbital (spin) orthogonal to $\xi(\sigma)$, respectively. The hopping matrix elements $t_{\mathrm{ij}}^{\xi \zeta}$ in the orbital basis $\{|x\rangle,|z\rangle\}$ with $|x\rangle \sim x^{2}-y^{2}$ and $|z\rangle \sim 3 z^{2}-r^{2}$ have the standard form [18]. The second term in Eq.(11) stands for Hund's coupling between electron spin $\mathbf{s}_{\mathbf{i}}=\sum_{\xi \sigma \sigma^{\prime}} \tilde{d}_{\mathbf{i} \xi \sigma}^{\dagger} \vec{\sigma}_{\sigma \sigma^{\prime}} \tilde{d}_{\mathbf{i} \xi \sigma^{\prime}}$ and the $t_{2 g}$ core spin $\mathbf{S}_{\mathbf{i}}^{\mathbf{c}}\left(S^{c}=3 / 2\right)$. The third term represents the AF superexchange $\left(J_{\mathrm{A} F}>0\right)$ between nearest-neighbor $t_{2 g}$ spins, while the fourth term is the inter-site Coulomb repulsion which favors checkerboard CO $(V>0)$. Finally

$$
H_{\mathrm{OO}}=2 \kappa^{\prime} \sum_{\left\langle\mathbf{j i j}^{\prime}\right\rangle}\left(1-n_{\mathbf{i}}\right) T_{\mathbf{j j}^{\prime}}
$$


accounts for the cooperative JT-interaction and describes the orbital-orbital (OO) interaction between further $\mathrm{Mn}^{3+}$ neighbors mediated by the $\mathrm{Mn}^{4+}$ ion inbetween [5] [Fig. [1 c)]. Hence, $\left\langle\mathbf{j i j}^{\prime}\right\rangle$ denotes three neighboring $\mathrm{Mn}^{3+}-\mathrm{Mn}^{4+}-\mathrm{Mn}^{3+}$ sites along $a$ or $b$ direction while the two-site orbital operator, $T_{\mathbf{j j}^{\prime}}$, in the $\{|x\rangle,|z\rangle\}$ basis has the form $T_{\mathbf{j j}^{\prime}}=T_{\mathbf{j}}^{z} T_{\mathbf{j}^{\prime}}^{z}+3 T_{\mathbf{j}^{x}}^{x} T_{\mathbf{j}^{\prime}}^{x} \mp \sqrt{3}\left(T_{\mathbf{j}}^{x} T_{\mathbf{j}^{\prime}}^{z}+\right.$ $\left.T_{\mathbf{j}^{z}}^{z} T_{\mathbf{j}^{\prime}}^{x}\right)$ expressed in terms of pseudospin operators: $T_{\mathbf{i}}^{z}=$ $\frac{1}{2} \sum_{\sigma}\left(n_{\mathbf{i} x \sigma}-n_{\mathbf{i} z \sigma}\right)$ and $T_{\mathbf{i}}^{x}=\frac{1}{2} \sum_{\sigma}\left(\tilde{d}_{\mathbf{i} x \sigma}^{\dagger} \tilde{d}_{\mathbf{i} z \sigma}+\tilde{d}_{\mathbf{i} z \sigma}^{\dagger} \tilde{d}_{\mathbf{i} x \sigma}\right)$. This term not only leads to the CE-type orbital order but also induces charge alternation on neighboring Mn sites. The interaction $H_{\mathrm{OO}}$ originates from a JT-driven displacement of the $\mathrm{Mn}^{4+}$ octahedra as observed in the CEphase of $\mathrm{La}_{1 / 2} \mathrm{Ca}_{1 / 2} \mathrm{MnO}_{3}$ 19]. The nearest-neighbor OO interaction $2 \kappa \sum T_{\mathbf{i j}}[20]$ is neglected here for simplicity as it does not contribute in the case of strong CO.

First we focus on the optical conductivity which is determined by the current-current correlation function

$$
\sigma(\omega)=\frac{1-e^{-\omega / T}}{N \omega} \operatorname{Re} \int_{0}^{\infty} d t e^{i \omega t}\left\langle j_{x}(t) j_{x}\right\rangle
$$

where the $x$-component of the current operator is $j_{x}=$ $i e \sum_{\langle\mathbf{i} \mathbf{j}\rangle} \sum_{\xi \zeta, \sigma} \sigma_{\mathbf{i j}}^{\xi \zeta} \delta_{x}^{\mathbf{i j}}\left(\tilde{d}_{\mathbf{i} \xi \sigma}^{\dagger} \tilde{d}_{\mathbf{j} \zeta \sigma}-\right.$ H.c. $)$ and $\delta_{x}^{\mathbf{i j}}$ denotes the $x$-component of the vector connecting sites $\mathbf{i}$ and $\mathbf{j}$. We evaluate this Kubo formula using the exact diagonalization technique for finite temperatures developed by Jaklič and Prelovšek 21] for cuprates and later used also for manganites [5, 18]. In order to make the problem numerically tractable, we simulated the core spins by $S^{c}=1 / 2$ spins and scale $J_{H}$ to preserve the splitting between high and low spin states. The data reported here was calculated for $J_{H}=15 t$. Although we exploited the translational symmetry to save storage and computation time, calculations were restricted to a small $\sqrt{8} \times \sqrt{8}$ cluster as only $S_{\text {tot }}^{z}=\sum_{\mathbf{i}} S_{\mathbf{i}}^{z}$ subspaces $\left(\mathbf{S}_{\mathbf{i}}=\mathbf{S}_{\mathbf{i}}{ }^{c}+\mathbf{S}_{\mathbf{i}}\right)$ can be treated separately, while a similar symmetry in the orbital sector is absent.

The subtle role of $H_{\mathrm{OO}}$ is to enhance CE type orbital polarization thereby supporting the $A F$ decoupling of FM-chains at low T (Fig. 1 1 ), while for small $\kappa^{\prime} \mathrm{DE}$ dominates in all directions leading to a FM charge and orbital ordered state [5]. In Fig. 2 we show the frequency and temperature dependence of $\sigma(\omega)$ emerging from ground states with AF-CE [Fig. 2(a)] and FM-CE [Fig. 2(b)] spin-orbital order, respectively. A characteristic feature of $\sigma(\omega)$ is a broad absorption maximum found for $2 t \lesssim \omega \lesssim 5 t$ which shifts towards lower frequencies by $\Delta \omega \simeq 0.5 t$ as temperature increases to $T \simeq 0.2 t$. Further increase of temperature leads to the melting of the orbital order and the closing of the charge gap $(\omega \lesssim 2 t)$ with further shift of the high frequency broad structure. The shape of $\sigma(\omega)$ and its temperature evolution is similar to the experimental data, e.g., for the cubic $\mathrm{La}_{0.5} \mathrm{Ca}_{0.5} \mathrm{MnO}_{3}$ 13] and layered $\mathrm{La}_{1 / 2} \mathrm{Sr}_{3 / 2} \mathrm{MnO}_{4}$ 11] compound. Assuming $t=0.4 \mathrm{eV}[18,20]$ one finds

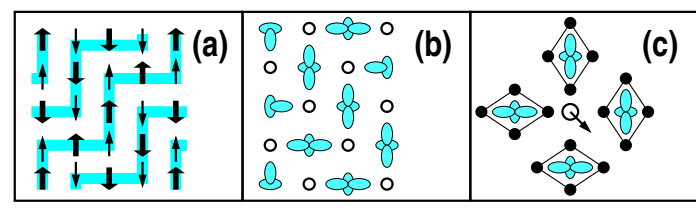

FIG. 1: (Color online) Sketch of the AF-CE spin, orbital, and lattice order. In (a) large (small) spins indicate sites with (without) an $e_{g}$ electron, respectively. Shading indicates direction of DE carrier propagation. In (b) full symbols represent occupied $e_{g}$ orbitals and in (c) full circles indicate the JT-shifted O ions, and the arrow the displacement of $\mathrm{Mn}^{4+}$.

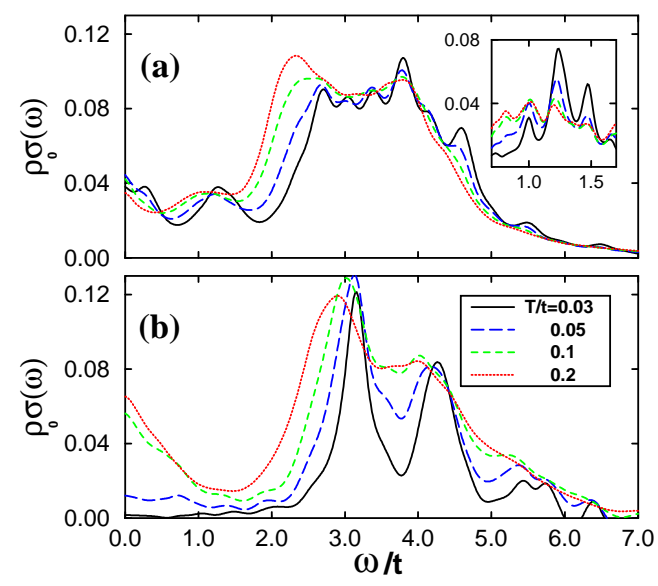

FIG. 2: (Color online) Temperature dependence of the optical conductivity $\sigma(\omega)$ obtained in the phase with: (a) AF-CE order in the ground state, and (b) a FM-CE ground state for $J_{\mathrm{A} F}=0.04 t$. Other parameters: (a) $V=0.2 t, \kappa^{\prime}=0.15 t$; (b) $V=t, \kappa^{\prime}=0.05 t$. The spectra are generated using a Lorentzian broadening $\Gamma=0.2 t$. Inset: Mid-gap absorption broadened with $\Gamma=0.05 t$.

the position of a broad peak structure in Fig. 2 centered close to the experimental value $1.2 \mathrm{eV}$ measured in singlelayer $\mathrm{La}_{1 / 2} \mathrm{Sr}_{3 / 2} \mathrm{MnO}_{4}$ [1]. A subtle test is the absolute scale of $\sigma(\omega)$ which is controlled by the sum rule that links $\sigma(\omega)$ and the kinetic energy of the model 18, 22. For the maximum of the absorption $\left(\sigma_{\max } \approx 0.1 \rho_{0}^{-1}\right)$ we find with $\rho_{0}=\hbar a / e^{2}$ [18] and the lattice constant $a \sim 5.5 \AA, \sigma_{\max } \approx 500(\Omega \mathrm{cm})^{-1}$. Observed values for $\sigma_{\text {max }}$ are $\sim 1000(\Omega c m)^{-1}$ in $\mathrm{La}_{1 / 2} \mathrm{Sr}_{3 / 2} \mathrm{MnO}_{4}$ 11, 12. and $\sim 700(\Omega \mathrm{cm})^{-1}$ in cubic $\mathrm{La}_{0.5} \mathrm{Ca}_{0.5} \mathrm{MnO}_{3}$ [13].

The main absorption in Fig. 2 describes excitations across the charge gap $\left(\Delta_{c} \simeq 3\left(V+2 \kappa^{\prime}\right)\right.$ in case of perfect $\mathrm{CO}$ ) which in the orbitally ordered state involve $e_{g}$ electron transfer mainly between bridge (ground state) and corner (excited state) sites within the same zig-zag chain. To understand the role of hopping $t$ it is useful to consider an embedded three-site model with an $e_{g}$ electron being either at the bridge $(|B\rangle)$ or at one of the corner $\left(\left|C_{L}\right\rangle,\left|C_{R}\right\rangle\right)$ sites. One finds the optical transition at $\omega_{c} \simeq \Delta_{c}+2 t^{2} / \Delta_{c}$, between bonding $\sim|B\rangle$ and non-bonding $\left(\left|C_{L}\right\rangle-\left|C_{R}\right\rangle\right) / \sqrt{2}$ states which have differ- 
ent parities. With increasing $T$ magnetic excitations are included in the thermodynamic average with reduced effective hopping, whose value is dictated by the DE mechanism for correlated $e_{g}$ electrons. Thus the reduction of the second order term $2 t^{2} / \Delta_{c}$ for these states results in a spectral shift towards lower $\omega_{c}$. Further reduction of $\omega_{c}$ is caused by melting of orbital and CO leading to gapless orbital excitations [18] and a reduction of $\Delta_{c}$.

In the FM spin state $\sigma(\omega)$ is characterized by two broad peaks and a well-developed charge gap [Fig. 2(b)]. Here, spin fluctuations are small and $\sigma(\omega)$ is dominated by charge-orbiton excitations, i.e., the pure charge excitation is well separated from two further peaks corresponding to the additional excitation of one and two orbitons. On the other hand, in the AF-CE spin ordered state spin fluctuations are substantial and inter-site charge transfer is accompanied by magnon and orbiton excitations leading to a broadened spectrum above the charge gap as well as to substantial in-gap absorption. The latter is completely absent in the FM state at low temperature.

To gain a deeper insight into the structure of $\sigma(\omega)$ in the CE spin-orbital phase, we have calculated [using a similar finite-temperature procedure as for $\sigma(\omega)]$ the dynamical spin, orbital, and spin-orbital response functions:

$$
A_{\mathbf{q}}(\omega)=\frac{1}{2 \pi} \int_{-\infty}^{\infty} d t e^{-i \omega t}\left\langle A_{\mathbf{q}}^{+} A_{-\mathbf{q}}^{-}(t)\right\rangle,
$$

where $A_{\mathbf{q}}=S_{\mathbf{q}}, T_{\mathbf{q}}$, or $C_{\mathbf{q}}$ stand for the spin, orbital, or combined spin-orbital operator $C_{\mathbf{q}}=\sum_{\mathbf{k}} S_{\mathbf{k}} T_{\mathbf{k}+\mathbf{q}}$, respectively. These response functions describe the simplest elementary excitations realized in the spin-orbital model. Strongly broadened collective spin excitations are present at low temperatures, but already at $T \simeq 0.1 t$ quasi-elastic scattering ( $\omega \simeq 0$ ) is found at all momenta (Fig. 3). The spin spectrum at low temperatures is strongly anisotropic, with pronounced precursors of Bragg peaks present at momenta $(\pi, 0)$ and $(\pi / 2, \pi / 2)$ at $\omega / t \simeq 0.05$ reflecting the spin pattern of the AFCE phase [see Fig. 1(a)]. Here, spins $S=1$ form two interpenetrating AF superlattices (with lattice constant $a=2$ ) which leads to the main maximum in $S_{\mathbf{q}}(\omega)$ at $\mathbf{q}=(\pi / 2, \pi / 2)$, while spins $S=1 / 2$ form one AF lattice in the frame rotated by 45 degrees leading to the smaller maximum in $S_{\mathbf{q}}(\omega)$ at $\mathbf{q}=(\pi, 0)$. Spin waves develop at higher energies $\omega \simeq 0.2 t$ due to the competition between AF $t_{2 g}$ - $t_{2 g}$ interactions and DE mechanism. An accurate assessment of the spin-wave dispersion would, however, require the study of larger clusters.

Orbital excitations are much more robust than the magnons and only weakly change with increasing temperature from $0.01 t$ to $0.1 t$. In the $T_{\mathbf{q}}(\omega)$ spectrum [see Fig. [4(a)-(b)] one finds a pronounced peak only at momentum $\mathbf{q}=(\pi / 2, \pi / 2)$ with $\omega \simeq 1.3 t$ at low temperatures which broadens with increasing temperature and becomes incoherent only for $T \gtrsim t$. Less coherent multi-

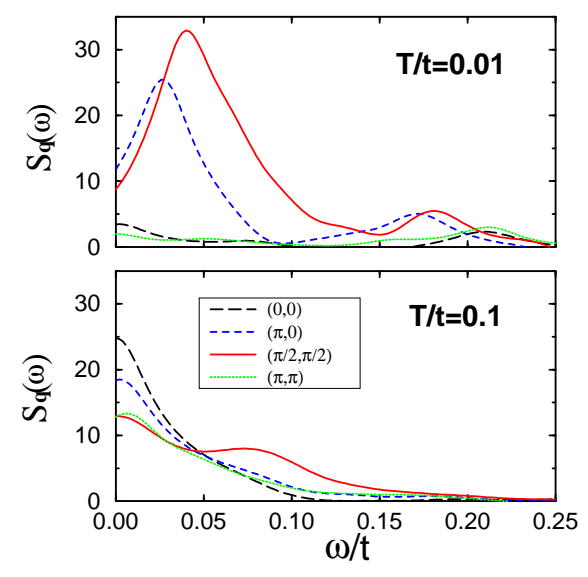

FIG. 3: (Color online) Dynamic spin structure factor $S_{\mathbf{q}}(\omega)$ in the AF-CE $(T=0.01 t)$ and paramagnetic $(T=0.1 t)$ phase, respectively. Parameters: $V=0.2, \kappa^{\prime}=0.15 t, J_{\mathrm{A} F}=0.04 t$, $J_{H}=15 t$. Spectra are broadened with $\Gamma=0.02 t$.

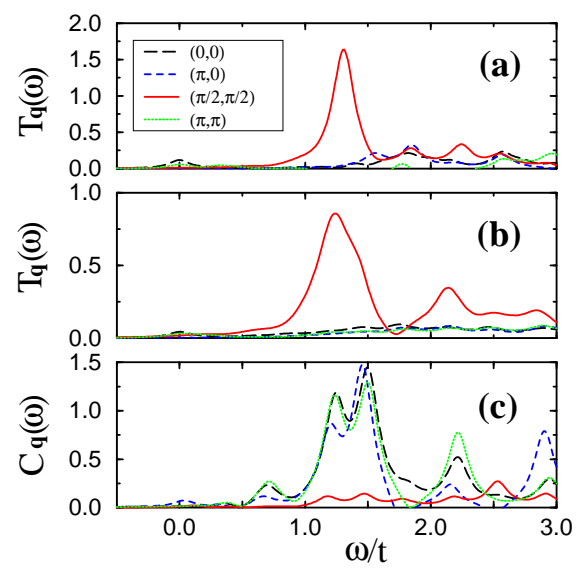

FIG. 4: (Color online) Dynamic structure factor in the $\{|x\rangle \pm$ $|z\rangle\}$ basis for orbital excitations $T_{\mathbf{q}}(\omega)$ (a) $T=0.01 t$, (b) $T=0.1 t$, and (c) combined spin-orbital excitations $C_{\mathbf{q}}(\omega)$ at $T=0.01 t$. Other parameters as in Fig. 3 and $\Gamma=0.1 t$.

peak structure is found at other momenta. The fact that the orbiton spectrum shows a pronounced peak only at $(\pi / 2, \pi / 2)$ momentum is a consequence of the pseudospin structure of the CE orbital phase [see Fig. [(b)] which have the same pattern as the $S=1$ spin structure [see Fig. 1(a)]. In the $\{|x\rangle \pm|z\rangle\}$ rotated orbital basis the OO term [see Eq. (2)] has Ising-like form, $T_{\mathbf{i j}} \simeq 3 T_{\mathbf{i}}^{z} T_{\mathbf{j}}^{z}$, and in the case of perfect $\mathrm{Mn}^{3+}-\mathrm{Mn}^{4+} \mathrm{CO}$ the energy of orbital excitation is estimated as $\omega_{\text {orb }} \simeq 12 \kappa^{\prime}$. Thus, for $\kappa^{\prime}=0.15 t$ one expects $\omega_{\text {orb }} \simeq 1.8 t$ while in our numerical results [see Fig. [4a)] the orbiton energy is reduced to $1.3 t$ due to imperfect $\mathrm{CO}$. Comparing the spectrum of orbital [Fig. 4(a)] and spin-orbital [Fig. 4(c)] excitations one finds that collective modes are formed as well. At energy $\omega \simeq 0.7 t$ and different momenta one finds a peak well below the orbital mode $(\omega \simeq 1.3 t)$ which 


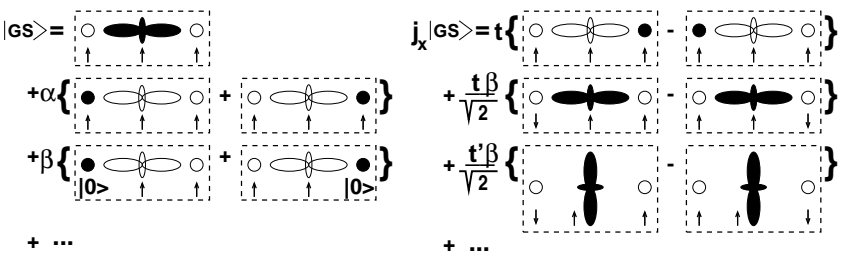

FIG. 5: A FM segment showing the dominant terms in the AF-CE ground state with $\mathrm{CO}(\alpha, \beta \ll 1)$ in the limit $J_{H} \rightarrow \infty$ (left). When applying the current operator $j_{x}$ (right), only the first term would contribute in the FM case. The second term $\sim \beta$ in $j_{x}|\mathrm{GS}\rangle$ (describing an orbital excitation) is finite due to quantum fluctuations in an AF environment. Here $t$ and $t^{\prime}\left(t>t^{\prime}\right)$ stand for two effective hoppings between corner and bridge sites, while full (empty) symbols stand for the sites occupied (unoccupied) by an $e_{g}$ electron with total spin (arrows) $S=1(S=1 / 2)$, and $|0\rangle \equiv\left|S=1, S^{z}=0\right\rangle$.

stands for a bound state [23, 24] while at $\omega \simeq 1.5 t \mathrm{a}$ strong maximum is found having a character of an antibound state. This indicates that the orbital excitations, although much more robust than the spin ones, play an active role also at a lower-energy scale. At higher energies $\omega \gtrsim 1.5 t$, however, $C_{\mathbf{q}}(\omega)$ and $T_{\mathbf{q}}(\omega)$ structure factors strongly differ.

A salient feature of $\sigma(\omega)$ is the in-gap absorption $\left(\omega<\Delta_{c}\right)$ in the AF-CE phase, Fig. 2(a), and the absence of such absorption for the FM-CE case in Fig. 2(b) at low temperature $(T \lesssim 0.1 t)$. We argue that the structure at $\omega \simeq t$ is an orbiton-like collective excitation which becomes infrared-active when parity is broken by AF corre-

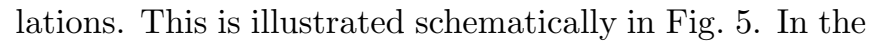
CO phase the ground state (GS) is dominated by configurations with occupied bridge sites but hybridized with states reached by inter-site hopping $(\sim t)$ or changed by core spin superexchange $\left(\sim J_{\mathrm{AF}}\right)$. As we deal with $d$ orbitals the hopping matrix has even parity leading to even GS as far as charge distribution is concerned. Consequently, the GS changed by the current operator is odd with respect to charge and (neglecting core spins) only processes across the optical gap are allowed (first line of $j_{x}|\mathrm{GS}\rangle$ in Fig. (5). In the presence of AF fluctuations these selection rules no longer strictly hold, as spin-exchange leads to an admixture of spin and spin-orbital excitations (second and third line of $j_{x}|\mathrm{GS}\rangle$ in Fig. (5) which do not cancel by symmetry.

This orbiton-magnon mechanism for the in-gap absorption is supported by the fact that the intensity of the structure in $\sigma(\omega)$ at $\omega \simeq t$ [see inset in Fig. 22(a)] increases (above the background of other excitations) with decreasing temperature in the spin-ordered case $(T \lesssim 0.1 t)$. The momentum conservation present in optical processes can be fulfilled combining $T_{\mathbf{q}}^{\dagger}$ and $S_{-\mathbf{q}}^{z}$ excitations with $\mathbf{q}=( \pm \pi / 2, \pm \pi / 2)$ which are dominant in the respective dynamical structure factors [see Fig. B(a) and Fig. [4(a)]. The decrease of the static structure factor
$S_{\mathbf{q}}=\int_{-\infty}^{\infty} d \omega S_{\mathbf{q}}(\omega)$ at $\mathbf{q}=( \pm \pi / 2, \pm \pi / 2)$ with temperature is apparently correlated with the suppression of the in-gap absorption in $\sigma(\omega)$.

In summary, optical conductivity and spin-orbital dynamics have been investigated in the CE phase of chargeordered manganites at quarter filling. Two contributions in our theory determine the charge gap: (i) elastic forces and (ii) Coulomb interaction. This is important to explain the recently discovered pico-second switching of optical spectra, which can be understood by the instantaneous reduction of the Coulomb contribution due to the suppression of CO by the ultra-short pulse, as well as the fast recovery after the pulse. We have shown that the cooperative JT coupling between further Mn neighbors, stabilizing the CE-type correlations in our model, can explain semi-quantitatively the optical data and its temperature dependence in half-doped manganites. Spin and orbital excitations lead to collective modes active in optical experiments, and are responsible for characteristic differences between AF- and FM CE phases.

We would like to thank A. M. Oleś for fruitful discussions. J. B. acknowledges the support of the MPI für Festkörperforschung, Stuttgart and by the Polish State Committee of Scientific Research (KBN) of Poland, Project No. 1 P03B 06826.

[1] Y. Tokura and N. Nagaosa, Science 288, 462 (2000).

[2] Y. Tokura, Physics Today 56, 50 (2003).

[3] J.B. Goodenough, Phys. Rev. 100, 564 (1955).

[4] D.I. Khomskii and K.I. Kugel, Phys. Rev. B 67, 134401 (2003); M.J. Calderon, A.J. Millis, and K.H. Ahn, ibid. 68, R100401 (2003).

[5] J. Bała, P. Horsch, and F. Mack, Phys. Rev. B 69, 094415 (2004).

[6] B.J. Sternlieb et al., Phys. Rev. Lett. 76, 2169 (1996).

[7] Y. Murakami et al., ibid. 80, 1932 (1998).

[8] M. Kubota et al., J. Phys. Soc. Jpn. 68, 2202 (1999).

[9] J. Geck et al., Phys. Rev. B 66, 184407 (2002).

[10] S. Mori, C. H. Chen, and S.-W. Cheong, Nature 392, 473 (1998).

[11] T. Ishikawa, K. Ookera, and Y. Tokura, Phys. Rev. B 59, 8367 (1999).

[12] J.H. Jung et al., Phys. Rev. B 61, 6902 (2000).

[13] K.H. Kim et al., Phys. Rev. Lett. 88, 167204 (2002).

[14] T. Ogasawara et al., J. Phys. Soc. Jpn. 71, 2380 (2002).

[15] I.V. Solovyev, Phys. Rev. B 63, 174406 (2001).

[16] M. Cuoco, C. Noce, and A.M. Oleś, ibid. 66, 094427 (2002).

[17] S. Yunoki, T. Hotta, and E. Dagotto, Phys. Rev. Lett. 84, 3714 (2000).

[18] F. Mack and P. Horsch, Phys. Rev. Lett. 82, 3160 (1999); P. Horsch et al., Phys. Rev. B 59, 6217 (1999).

[19] P. G. Radaelli et al., Phys. Rev. B 55, 3015 (1997).

[20] L.F. Feiner and A.M. Oleś, Phys. Rev. B 59, 3295 (1999).

[21] J. Jaklič and P. Prelovšek, Adv. Phys. 49, 1 (2000).

[22] M. Aichhorn et al., Phys. Rev. 65, 201101 (2002).

[23] J. van den Brink et al., Phys. Rev. B 58, 10276 (1998). 
[24] J. Bała, A.M. Oleś, and G.A. Sawatzky ibid. 63, 134410 (2001). 